\title{
Brief memory reactivations induce learning in the numeric domain
}

Gilad Schrift ${ }^{1}$, Dror Dotan ${ }^{2}$, Nitzan Censor ${ }^{1}$

1. School of Psychological Sciences and Sagol School of Neuroscience, Tel Aviv University, Tel Aviv 69978, Israel

2. School of Education and Sagol School of Neuroscience, Tel Aviv University, Tel Aviv 69978, Israel

* Address of correspondence: censornitzan@ @ tauex.tau.ac.il, Sharet Building, School of Psychological Sciences, Tel Aviv University, Tel Aviv 69978, Israel.

Declaration of Interests: The authors declare no competing interests.

Keywords: numeric learning; skill memory; memory reactivation; 


\begin{abstract}
Learning of arithmetic facts such as the multiplication table requires time-consuming, repeated practice. In light of evidence indicating that reactivation of encoded memories can modulate learning and memory processes at the synaptic, system and behavioral levels, we asked whether brief memory reactivations can induce human learning in the numeric domain. Adult participants performed a novel number-fact retrieval task in which they learned arbitrary numeric facts. Following encoding and a baseline test, 3 passive, brief reactivation sessions of only 40 seconds each were conducted on separate days. Learning was evaluated in a retest session. Results showed reactivations induced learning, with improved performance at retest relative to baseline test. Furthermore, performance was superior compared to a control group performing test-retest sessions without reactivations, who showed significant memory deterioration. A standard practice group completed activeretrieval sessions on 3 separate days, and showed significant learning gains. Interestingly, while these gains were higher than those of the reactivations group, subjects showing reactivation-induced learning were characterized by superior efficiency relative to standard practice subjects, with higher rate of improvement per practice time. A follow-up long-term retention experiment showed that 30 days following initial practice, weekly brief reactivations reduced forgetting, with participants performing superior to controls undergoing the same initial practice without reactivations. Overall, the results demonstrate that brief passive reactivations induce efficient learning and reduce forgetting within a numerical context. Time-efficient practice in the numeric domain carries implications for enhancement of learning strategies in daily-life settings, including in learning disorders caused by neurological conditions.
\end{abstract}




\section{Introduction}

Throughout a wide range of research disciplines extending from cognitive neuroscience to neurorehabilitation and daily-life activities, the prevailing dogma is that learning is enabled by extensive skill execution, known as 'practice makes perfect' ${ }^{1,2}$. For instance, numerous studies have shown a significant relationship between the time that students spend in learning and their mathematical performance ${ }^{3,4}$. This dogma as a unitary route for learning has been recently challenged by behavioral studies showing that procedural skill learning can be achieved without extensive exercise but rather via brief reactivations of the learned $\operatorname{task}^{5,6}$. At the neural level, it has been shown that distinct content-sensitive patterns of neural activity that occur during a perceptual experience, recur when the experience is reminded ${ }^{7-9}$ and predict the consequential memory retrieval ${ }^{9-16}$.

Potentially related to memory-reactivation learning is the phenomenon of memory reconsolidation ${ }^{17-19}$. As stabilized memories are reactivated, either by retrieval or by a reminder cue, they revert to a labile state. Reconsolidation is the process that follows in which these memories are re-stabilized and are susceptible to modifications by incoming inputs. Accumulating evidence indicates that these existing memories can be reinforced, degraded, or updated by the inclusion of new information during the offline reconsolidation phase ${ }^{5,6,16-29}$. With research originating from rodents fear-conditioning frameworks extending to human skill learning, evidence supporting the dynamics of the reconsolidation phase now subsists at the synaptic, systems and behavioral levels.

Here, we set to investigate whether memory-reactivations can induce learning in dailylife settings. To address this question, we embedded the framework of memory reactivations into the field of numerical cognition, specifically that of numeric facts learning.

The ability to recall basic arithmetic facts in a precise way with little effort is often referred to as fluency or automaticity ${ }^{30}$. Findings from research of mathematical education show the benefits of achieving fluency in basic mathematical facts ${ }^{30-34}$, which may be essential in order to develop estimation and mental computation skills ${ }^{35-39}$. However, many students show substantial individual differences and have difficulties in achieving fluency in basic arithmetic facts retrieval, such as multiplication facts ${ }^{35,36,40-42}$. Therefore, if reactivation-induced learning indeed enables improved fluency, it could prove as a time efficient type of practice in the numeric domain and carry potential implications for daily-life practice strategies, including in learning disorders caused by neurological conditions. 
To evaluate the extent of numeric facts learning by memory-reactivations in a population which is already familiar with elementary arithmetic, we used a novel number-fact retrieval task. In this task, participants had to learn 8 novel numeric facts generated according to specific principles (see Methods), and later recalled them as quickly and accurately as they can. The facts were first taught and then tested in an initial encoding-test session, with a retest session conducted 8 days later (Figure 1a). Participants in the Reactivations group performed brief reactivations over three separate days between encoding-test and retest. Each of these reactivation sessions lasted merely 40 seconds, in which participants' memory was passively reactivated briefly by a presentation of the facts, in both auditory and visual modalities. The Control group performed the encoding-test and retest sessions, without memory reactivations. Participants in the Standard Practice group performed three activeretrieval practice sessions between encoding-test and retest, each similar to the day 1 encoding session. Learning gains were quantified as the difference in performance between the baseline test and the retest sessions. In an additional experiment, we examined whether such a form of rapid learning with brief exposures can enable efficient long-term retention following initial standard problem-solving practice.

\section{Methods}

\section{Subjects}

135 naive healthy participants, ages $18-40$ years (113 female; mean age $24.0 \pm 2.2$ s.d.) gave their written informed consent to participate in the project, which was approved by Tel Aviv University's Ethics committee. All procedures were in accordance with approved guidelines. Sample sizes were estimated based on power analysis of pilot studies conducted in the lab, using $\mathrm{G}^{*}$ Power $3^{43,44}$. Participants were randomly allocated to experimental conditions, which were conducted in a single-blinded fashion. Participants were required to sleep at least 6 hours the night before each experimental session, which was performed during daytime. All sessions were conducted online using Pavlovia (https://pavlovia.org/). Seven participants from the first experiment (2 Reactivations, 4 Control, 1 Standard Practice) and two participants from the retention experiment (2 Control) whose test-retest learning was 1.5 times the interquartile range above the third quartile or below the first quartile, were identified as outliers and excluded from further analysis ${ }^{45}$. 


\section{Number-Fact Retrieval Task}

To mimic numeric fact learning within a population of adult participants, who are already familiar with elementary arithmetic, we generated 8 novel numeric facts. In all facts, the operands were single digits and the result was a 2-digit number (which could be prime), with no repeating digit within a fact. Only the digits 2-9 were used. When generating facts, we aimed for no obvious relation between the operands and the results of a fact; and for low between-fact similarity that may disrupt learning ${ }^{46,47}$. The facts were presented using the nonexistent operator '\&' (e.g., “3 \& $8=29$ ”). All participants learned the same 8 facts. Performance was quantified by the recall score: a correct result digit in the correct position of the result scored 1 point, and each correct digit in the incorrect position scored 0.5 points. Thus, the score for each fact was between 0 and 2. To calculate recall score in a scale of 0 100 , the total points was then divided by 16 , the maximum possible number of points. Recall response times were also measured. The task was created using PsychoJs ${ }^{48}$.

\section{Experimental procedure}

Day 1 Session, Encoding - Memory was initially encoded and consolidated in the Day 1 session, in which the facts were first presented and taught. The session was identical for participants in all groups. Participants were first given instructions on how the task interface works and completed a warm-up exercise $(0 \& 0=0)$.

They then performed 3 rounds of active-retrieval practice. Each round consisted of a presentation stage followed by a recall stage. In the presentation stage, each item was presented on the screen for 6 seconds and was simultaneously narrated aloud. In the recall stage, the exercises without the results were presented (visually and auditory) one by one, and the participants were asked to fill-in the result as accurately and quickly as they could within a time limit of 8 seconds. The correct result was then shown, i.e., feedback was provided for both incorrect and correct responses. The order of facts was random both in presentation and in recall.

After a 5-minute break, participants completed a forced-choice test in which they were required to choose, for each of the 8 facts, the correct result out of 4 options with no feedback provided. Options consisted of the correct result, a distractor containing one digit from the correct result in the correct position and one irrelevant digit, a distractor containing one digit from the correct result in the incorrect position and one irrelevant digit, and a 
distractor which was the result of a different fact. The forced-choice test was included in order to determine if additional training was required for better encoding, and repeated up to 2 times. An additional practice round was given for facts unsuccessfully recalled in the forced-choice test (Figure 1b).

Day 1 Session, Test - Following a 30-minute break, a free recall baseline measurement was collected, in which the facts were shown in random order, without feedback. In addition, a forced-choice test was administered as described above; the distractors were selected using the same method, but the specific distractors were different from the ones used earlier in the session. After each forced-choice question, participants also rated their subjective confidence in their answer being correct on a visual analog scale of one to seven (Figure 1c).

Day 2-4 Sessions, Practice - The Reactivations group $(\mathrm{n}=28)$ sessions included a passive, five second visual-auditory single presentation of each fact in random order, thus each reactivation session was only 40 seconds in total (Figure 1d). The Control group $(n=31)$ performed only test and retest sessions, without reactivations. The Standard Practice group's $(n=30)$ procedure was identical to the first part of the Day 1 active-retrieval session: it consisted of the 3 practice rounds, but did not include the forced-choice test, the additional practice rounds and the post-practice retest.

Day 5 Session, Retest - Participants returned for a retest session, which was the same as the baseline-test in the $1^{\text {st }}$ day, with different distractors in the forced-choice questions (Figure 1c).

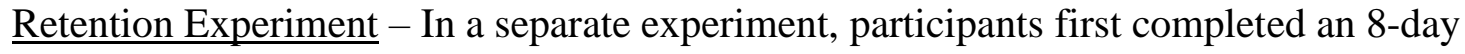
standard practice procedure and were tested for retention following 30 days (Figure 3a). Participants were divided into 2 groups: A Reactivations group $(n=19)$ who completed 40second reactivations once per week between the post-training retest and the long-term retention test, and a Control group $(\mathrm{n}=18)$ without reactivations. Weekly reactivations were identical to the reactivations in the first experiment. The retention test was similar to the pretraining test and post-training retest, with different distractors for the forced-choice questions. 
a

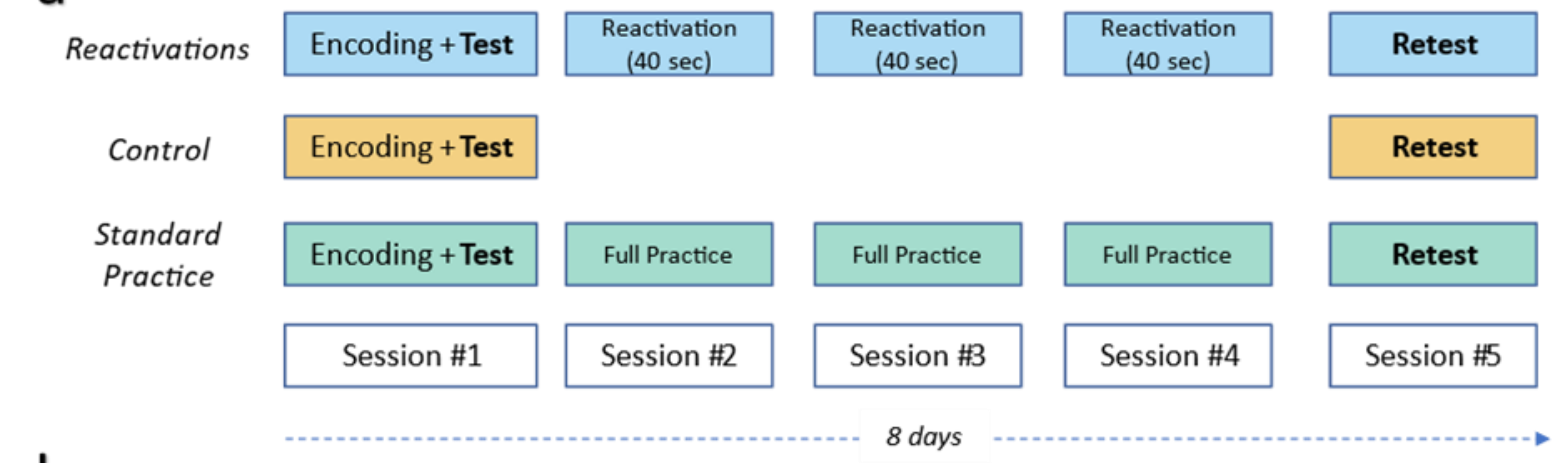

b Encoding
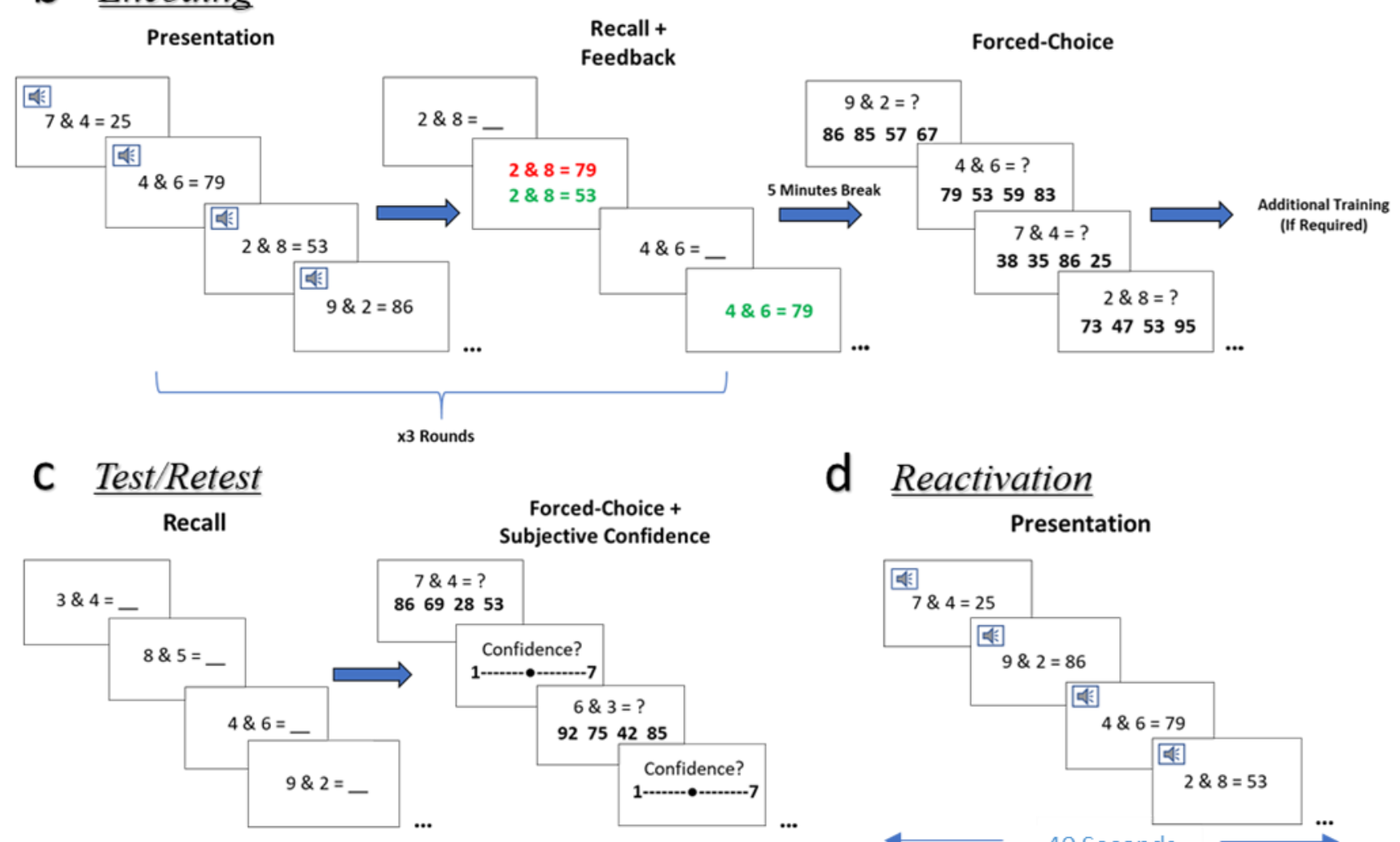

d Reactivation

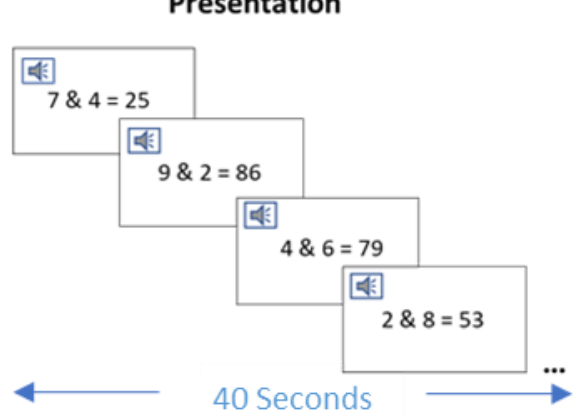

Figure 1: Number-fact retrieval task. (a) Experimental design. Participants first encoded the facts and were tested for baseline performance following 30 minutes. Participants in the Reactivations group performed 3 brief reactivation sessions in which their memory was briefly reactivated for only 40 seconds of passive facts presentation. The Control group performed the test and retest sessions without reactivations. Participants in the Standard Practice group performed 3 full practice sessions between test and retest, each containing 3 rounds of facts presentation and recall with feedback. Retest sessions were conducted 8 days following the $1^{\text {st }}$ session. (b) Encoding Session: included 3 rounds of active-retrieval practice. Each round consisted of a presentation stage and a recall stage with feedback (correct response $=$ green, wrong response $=$ red accompanied with the correct answer). Participants were asked to fill-in the result as accurately and as fast as they can within a time limit of 8 seconds. Following a 5-minute break, they solved each fact as a 4-alternative forced-choice question in order to determine if an additional training round is required for efficient encoding. Accordingly, an additional practice round was performed for facts with incorrect forced-choice answers. (c) Test/Retest Session: consisted of a free recall round, followed by a forced-choice questions round. After each forced-choice question, participants rated their subjective confidence in their answer. (d) Reactivation Session: consisted of five seconds of a visual-auditory presentation of each fact in random order, for a total of 40 seconds. 


\section{Results}

To verify that there were no baseline differences between groups, we conducted a one-way ANOVA of the baseline test sessions. No group effect was found on the participants' recall score $\left(\mathrm{F}_{2,86}=0.135, P=0.87\right)$, reaction times $(\mathrm{RTs})\left(\mathrm{F}_{2,86}=1.083, P=\right.$ $0.34)$, forced choice $(\mathrm{FC})$ correct answers $\left(\mathrm{F}_{2,86}=0.219, P=0.80\right)$ or level of confidence $\left(\mathrm{F}_{2,86}=1.196, P=0.31\right)$. We then continued to test whether the brief reactivations induced learning gains. A repeated measures ANOVA on the participants' score, with Session (baseline, test) and Group (Reactivations, Control, Standard Practice) as factors, showed significant Session $\left(\mathrm{F}_{1,86}=10.518, P=0.002\right)$ and Session $\mathrm{x}$ Group interaction effects $\left(\mathrm{F}_{2,86}=\right.$ 52.413, $\left.P<10^{-14}\right)$. Post-hoc paired-samples t-tests verified that the Reactivations group obtained significant learning gains (mean test-retest score change $=8.48 \pm 2.94$ S.E., $\mathrm{t}(27)=$ $2.89, P=0.008$ ), in contrast to the Control group (Post-hoc Tukey test, $P<10^{-6}$, Bonferroni corrected) whose performance deteriorated (mean score change $=-18.04 \pm 3.15$ S.E., $\mathrm{t}(30)=$ $-5.72, P<10^{-5}$ ) (Figure 2a,b). The Standard Practice group showed the largest learning gains (mean score change $=27.71 \pm 3.39$ S.E., $\mathrm{t}(29)=8.18, \mathrm{P}<10^{-8}$ ). While learning gains of the Standard Practice group were considerably higher than those of the Reactivations group (Post-hoc Tukey test, $P=0.0001$, Bonferroni corrected), a one-way ANOVA between groups examining the practice efficiency - meaning the rate of improvement per practice time, revealed that subjects who exhibited reactivation-induced learning $(n=21)$ were characterized by superior efficiency relative to the standard group subjects who showed learning $\left(\mathrm{n}=29 ; \mathrm{F}_{1,48}=22.73, P=0.00002\right)$ (Figure 2c).

A repeated measures ANOVA on the participants' RTs showed a significant Session $\left(\mathrm{F}_{1,86}=14.613, P=0.0003\right)$ and Session $\mathrm{x}$ Group interaction effects $\left(\mathrm{F}_{2,86}=7.688, P=\right.$ 0.0009). Paired-samples t-tests tests revealed that the Reactivations group had a significant reduction in reaction times (mean test-retest RT change $=-487 \mathrm{~ms} \pm 174 \mathrm{~ms} \mathrm{S.E.,} \mathrm{t}(27)=-$ 2.80, $P=0.009$ ), while the Control group did not (mean change $=0.124 \mathrm{~ms} \pm 177 \mathrm{~ms} \mathrm{~S}$.E, $\mathrm{t}(30)=0.70, P=0.49)$. RT reduction was also observed for the Standard Practice group (mean change $=-867 \mathrm{~ms} \pm 194 \mathrm{~ms} \mathrm{~S} . \mathrm{E}, \mathrm{t}(29)=-4.47, P=0.0001)$. 

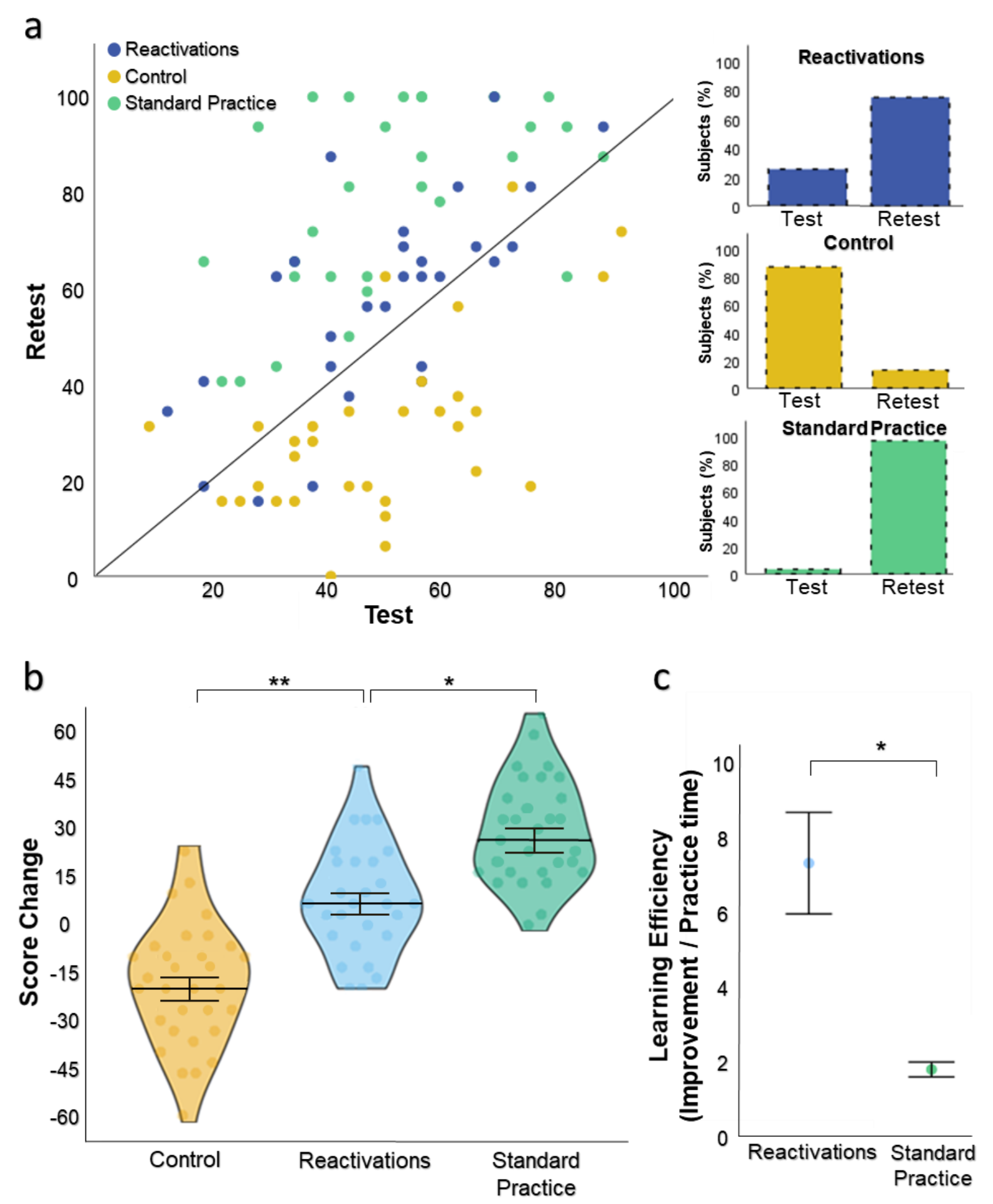

Figure 2: Reactivation-induced learning gains. (a) Baseline test versus retest single-subject performance score, presented in a scatterplot along a unit slope line $(y=x)$, where each point reflects a participant. Data accumulating above the unit line reflect participants who improved from test to retest, expressing learning gains, while data points below the line indicate degraded retest performance. Colored bars reflect the percentage of participants on each side of the unit slope line. (b) Violin graph showing the mean score change from test to retest sessions. Each point reflects a participant. (c) Subjects showing reactivation-induced learning were characterized by superior learning efficiency relative to Standard Practice subjects showing learning, with higher rate of improvement per practice time in minutes. $* P<0.0002$, $* * P<10^{-6}$. Error bars represent standard error of the mean. 
A repeated measures ANOVA on FC performance also showed a significant Session $x$ Group interaction (FC correct answers: $\mathrm{F}_{2,86}=46.483, P<10^{-13}$; FC confidence: $\mathrm{F}_{2,86}=$ 26.496, $P<10^{-8}$ ). Paired-samples t-tests revealed that the Reactivations group maintained their number of FC correct answers and confidence (mean test-retest FC correct answers change $=0.429 \pm 0.24$ S.E., $\mathrm{t}(27)=1.76, P=0.09$; confidence change $=0.31 \pm 0.18$ S.E., $\mathrm{t}(27)=1.71, P=0.1)$ while the Control group showed significant deterioration (correct answers change $=-1.96 \pm 0.27$ S.E., $\mathrm{t}(30)=-7.33, P<10^{-7}$; confidence change $=-1.02 \pm$ 0.18 S.E, $\left.\mathrm{t}(30)=-5.74, P<10^{-5}\right)$. The Standard Practice group showed improvement in FC performance and confidence $\left(\mathrm{FC}\right.$ change $=1.47 \pm 0.25 \mathrm{~S} . \mathrm{E}, \mathrm{t}(29)=5.81, P<10^{-5}$; confidence change $=1.01 \pm 0.24$ S.E, $\mathrm{t}(29)=4.24, P=0.0002)$.

In sum, the results indicate that brief reactivations induced significant learning gains and prevented memory deterioration, which was significant in the absence of reactivations. In addition, while learning gains were higher following standard practice, subjects who benefited from reactivation-induced learning were characterized by superior efficiency relative to standard practice subjects, with higher rate of improvement per practice time.

The present experiment was performed over a period of 8 days. To examine the scope of the reactivations effect, in particular whether reactivations would be beneficial also for long-term retention (typical for real-life situations of memorizing arithmetic facts), we conducted an additional experiment which tested reactivations over a longer period of time. In this experiment, participants first completed an 8-day (5 sessions) standard practice procedure, and were then divided into 2 groups with a retention test performed following 30days. The Reactivations group underwent a 40-second reactivation once per week between post-training retest and the retention test, while retention for the Control group was tested without reactivations during the retention interval (Figure 3a).

As expected, a repeated measures ANOVA on the initial training course with Session (Pre-training test, Post-training retest) and Group (Reactivations, Control) as factors showed no group effect on recall score $\left(\mathrm{F}_{1,35}=0.705, P=0.41\right)$, $\mathrm{RTs}\left(\mathrm{F}_{1,35}=1.059, P=\right.$ $0.31)$, FC performance $\left(\mathrm{F}_{1,35}=0.271, P=0.61\right)$, and $\mathrm{FC}$ confidence $\left(\mathrm{F}_{1,35}=0.033, P=0.86\right)$. We then continued to test whether the brief reactivations promoted long-term memory retention. A repeated measures ANOVA on participants' score with Session (Post-training retest, Retention) and Group (Reactivations, Control) as factors revealed a significant effect of Session $\left(\mathrm{F}_{1,35}=39.91, P<10^{-6}\right)$. Importantly, a significant Session x Group interaction $\left(\mathrm{F}_{1,35}=6.26, P=0.017\right)$ indicated that the decline in performance in the Reactivations group 
(mean post-training retest - retention score change $=-12.99 \pm 3.48$ S.E., $\mathrm{t}(18)=-3.72, P=$ 0.002 , paired t-test) was smaller than in the Control group (mean score change $=-30.03 \pm$ 5.95 S.E., $\mathrm{t}(17)=-5.05, P=0.0001$, paired t-test), suggesting that weekly reactivations reduced forgetting (Figure 3b,c).

No significant Session x Group interactions were observed on RTs, FC $\left(\mathrm{F}_{1,35}=0.286\right.$, $\left.P=0.60 ; \mathrm{F}_{1,35}=0.722, P=0.40\right)$, and a significant interaction was observed on $\mathrm{FC}$ confidence $\left(\mathrm{F}_{1,35}=4.213, P=0.048\right)$.

a

\begin{tabular}{|c|c|c|c|c|c|c|c|}
\hline Reactivations & Test & Practice Retest & $\begin{array}{c}\text { Reactivation } \\
(40 \mathrm{sec})\end{array}$ & $\begin{array}{l}\text { Reactivation } \\
\text { (40 sec) }\end{array}$ & $\begin{array}{l}\text { Reactivation } \\
(40 \mathrm{sec})\end{array}$ & $\begin{array}{l}\text { Reactivation } \\
(40 \mathrm{sec})\end{array}$ & Retention Test \\
\hline Control & Test & Practice Retest & & & & & Retention Test \\
\hline
\end{tabular}

b
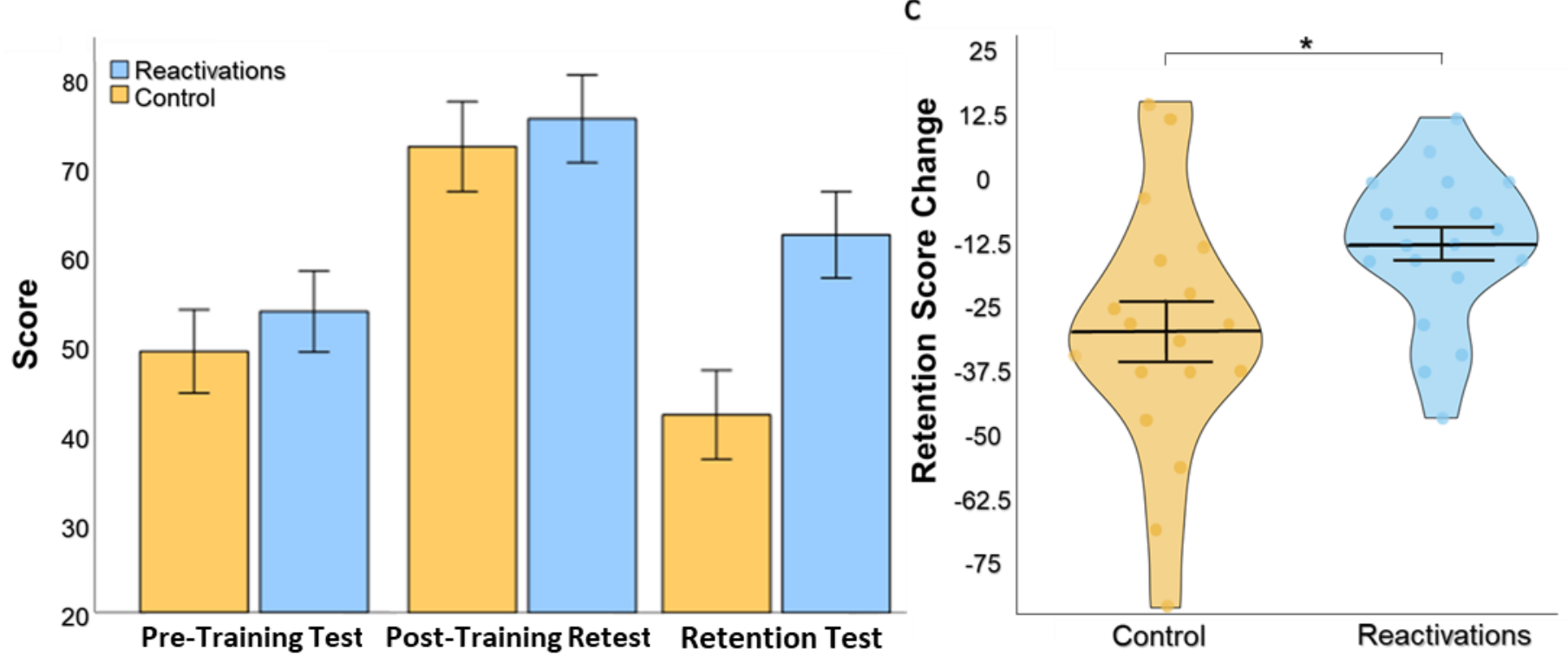

Figure 3: Long-term retention (a) Experimental design. Participants first completed an 8-day standard practice procedure and were tested for retention following 30 days. Participants were divided into 2 groups: Reactivations which completed 40-second reactivation sessions once per week between post-training retest and retention test, and Controls without reactivations. (b) Mean scores per session for each group. (c) Violin graph showing the mean score change from post-training retest to retention. Each point reflects a participant. $* P<0.02$. Error bars represent standard error of the mean. 


\section{Discussion}

In this study, we aimed to reveal whether reactivating a consolidated memory of numeric facts can strengthen it and promote learning gains. The results showed that 40 -second visualauditory reactivations improved recall of the numeric facts, measured 8 days following encoding. Reactivation-induced learning resulted in a significant improvement compared to the baseline test, and was superior to control without reactivations which showed deteriorated performance relative to baseline. Furthermore, while average learning gains induced by reactivations were lower than the standard practice group, subjects showing reactivation-induced learning were characterized by superior efficiency relative to standard practice subjects, with higher rate of improvement per practice time. This efficiency of learning in the reactivations group relative to the standard practice group may be of crucial importance for real-life pedagogical settings. We then initiated an additional experiment, examining whether reactivations can prove beneficial for long-term retention, which is most relevant for real-life learning. The results showed that brief memory reactivations performed only once per week during 4 weeks following the initial learning phase, reduced long-term forgetting of numeric facts compared to controls.

These findings are in good agreement with an increasing number of studies reporting memory strengthening via reactivations in procedural learning ${ }^{5,6}$. Taken together with experiments showing memory interference effects induced by memory reactivation ${ }^{49-53}$, our findings support the assumption that the reactivation process facilitates learning associated with declarative human memory, and exists also in a mathematical context. The neural mechanisms underlying this novel form of reactivation-induced learning remain to be determined. Recent research points to the existence of an overlapping fronto-parietal network of numerical cognition composed of intraparietal and prefrontal areas ${ }^{54-56}$. This network includes nodes related to the three primary representational codes which exist for numbers according to the prominent Triple Code Model: the visual Arabic number form (e.g., "2"), the auditory verbal word (e.g., "two"), and analogue non-symbolic size

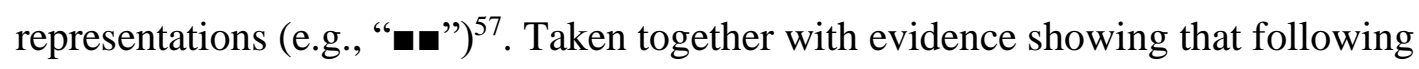
encoding, memories with more neural reactivations during awake rest periods are better remembered $^{17}$, it is conceivable that neural reactivations within this network play a role in the consolidation of hippocampus-dependent memories ${ }^{58}$, and subsequently facilitate reactivation-induced learning of numerical facts. In addition, future studies should test whether integrating active-retrieval testing in the process of reactivations or other 
combinations of regular practice and reactivations, would result in even more efficient learning processes ${ }^{17,59,60}$.

In sum, our findings in two separate experiments demonstrate that memoryreactivations, induced by brief audio-visual presentations, can lead to efficient learning and reduced forgetting of numerical facts. This unique form of skill learning approach may also help promote strategies for developing innovative, productive learning practices for numerical fluency, providing a time-efficient tool for skill acquisition, relevant for daily-life educational settings. Additional research may also reveal whether this form of learning can facilitate implementation of novel learning strategies geared to reduce the requirement for prolonged task execution, potentially beneficial for disorders caused by neurological conditions such as ADHD.

\section{Acknowledgements}

The study was supported by the Israel Science Foundation (ISF 526/17) and the European Research Council (ERC-2019-COG 866093). D.D received support from the Jacobs Foundation (grant no. 2019-1320-05).

\section{Data Availability}

The datasets collected and analyzed during the current study are available upon request from the corresponding author.

\section{Author Contributions}

Conceptualization, G.S., D.D. and N.C.; Methodology, G.S., D.D. and N.C.; Data Collection and Analysis, G.S.; Writing and Editing, G.S., D.D. and N.C.;

\section{References}

1. Karni, A. \& Sagi, D. The time course of learning a visual skill. Nature, 365, 250-252 (1993).

2. Censor, N., Sagi, D. \& Cohen, L. G. Common mechanisms of human perceptual and motor learning. Nature Reviews Neuroscience, 13, 658-664 (2012). 
3. Gettinger, M. Effects of maximizing time spent and minimizing time needed for learning on pupil achievement. American educational research journal, 26(1), 73-91 (1989).

4. Fisher, C. W., Berliner, D. C., Filby, N. N., Marliave, R., Cahen, L. S., \& Dishaw, M. M. Teaching behaviors, academic learning time, and student achievement: An overview. The Journal of classroom interaction, 17(1), 2-15( 1981).

5. Amar-Halpert, R., Laor-Maayany, R., Nemni, S., Rosenblatt, J. D. \& Censor, N. Memory reactivation improves visual perception. Nature Neuroscience, 20, 13251328 (2017).

6. Herszage, J., Sharon, H. \& Censor, N. Reactivation-induced motor skill learning. Proc. Natl. Acad. Sci., 118, 2102242118 (2021).

7. Ben-Yakov, A., Dudai, Y. \& Mayford, M. R. Memory retrieval in mice and men. Cold Spring Harb. Perspect. Biol., 7, a021790 (2015).

8. Favila, S. E., Lee, H. \& Kuhl, B. A. Transforming the Concept of Memory Reactivation. Trends in Neurosciences, 43, 939-950 (2020).

9. Ritchey, M., Wing, E. A., LaBar, K. S. \& Cabeza, R. Neural similarity between encoding and retrieval is related to memory via hippocampal interactions. Cereb. Cortex, 23, 2818-2828 (2013).

10. Schapiro, A. C., McDevitt, E. A., Rogers, T. T., Mednick, S. C. \& Norman, K. A. Human hippocampal replay during rest prioritizes weakly learned information and predicts memory performance. Nat. Commun., 9, 1963-1966 (2018).

11. Staresina, B. P., Alink, A., Kriegeskorte, N. \& Henson, R. N. Awake reactivation predicts memory in humans. Proc. Natl. Acad. Sci., 110, 21159-21164 (2013).

12. Polyn, S. M., Natu, V. S., Cohen, J. D. \& Norman, K. A. Category-specific cortical activity precedes retrieval during memory search. Science, 310, 1963-1966 (2005).

13. St-Laurent, M., Abdi, H. \& Buchsbaum, B. R. Distributed patterns of reactivation predict vividness of recollection. J. Cogn. Neurosci., 27, 2000-2018 (2015).

14. Lee, H., Samide, R., Richter, F. R. \& Kuhl, B. A. Decomposing Parietal Memory Reactivation to Predict Consequences of Remembering. Cereb. Cortex, 29, 33053318 (2019). 
15. Kuhl, B. A., Bainbridge, W. A. \& Chun, M. M. Neural reactivation reveals mechanisms for updating memory. J. Neurosci., 32, 3453-3461 (2012).

16. Staresina, B. P., Cooper, E. \& Henson, R. N. Reversible information flow across the medial temporal lobe: The hippocampus links cortical modules during memory retrieval. J. Neurosci., 33, 14184-14192 (2013).

17. Nader, K. \& Hardt, O. A single standard for memory: The case for reconsolidation. Nature Reviews Neuroscience, 10 224-234 (2009).

18. Alberini, C. M. \& Ledoux, J. E. Memory reconsolidation. Current Biology, 23 746750 (2013).

19. Nader, K., Schafe, G. E. \& Le Doux, J. E. Fear memories require protein synthesis in the amygdala for reconsolidation after retrieval. Nature, 406, 722-726 (2000).

20. Suzuki, A., Josselyn, S. A., Frankland, P. W., Masushige, S., Silva, A. J., \& Kida, S. Memory reconsolidation and extinction have distinct temporal and biochemical signatures. Journal of Neuroscience, 24(20), 4787-4795 (2004).

21. Lee, J. L. Memory reconsolidation mediates the strengthening of memories by additional learning. Nature Neuroscience, 11(11), 1264-1266 (2008).

22. Shema, R., Haramati, S., Ron, S., Hazvi, S., Chen, A., Sacktor, T. C., \& Dudai, Y. Enhancement of consolidated long-term memory by overexpression of protein kinase $\mathrm{M} \zeta$ in the neocortex. Science, 331, 1207-1210 (2011).

23. Robertson, E. M. New Insights in Human Memory Interference and Consolidation. Curr. Biol., 22, 66-71 (2012).

24. Censor, N., Horovitz, S. G. \& Cohen, L. G. Interference with Existing Memories Alters Offline Intrinsic Functional Brain Connectivity. Neuron, 81, 69-76 (2014).

25. Lugassy, D., Herszage, J., Pilo, R., Brosh, T. \& Censor, N. Consolidation of complex motor skill learning: evidence for a delayed offline process. Sleep, 41, 1-7 (2018).

26. Walker, M. P., Brakefield, T., Hobson, J. A. \& Stickgold, R. Dissociable stages of human memory consolidation and reconsolidation. Nature, 425, 616-620 (2003).

27. Schiller, D., Kanen, J. W., LeDoux, J. E., Monfils, M. H. \& Phelps, E. A. Extinction during reconsolidation of threat memory diminishes prefrontal cortex involvement. 
Proc. Natl. Acad. Sci., 110, 20040-20045 (2013).

28. Herszage, J. \& Censor, N. Memory Reactivation Enables Long-Term Prevention of Interference. Curr. Biol., 27, 1529-1534.e2 (2017).

29. Censor, N., Buch, E. R., Nader, K. \& Cohen, L. G. Altered Human Memory Modification in the Presence of Normal Consolidation. Cereb. Cortex, 26, 3828 (2016).

30. Hasselbring, T. S., Goin, L. I. \& Bransford, J. D. Developing Math Automatically in Learning Handicapped Children: The Role of Computerized Drill and Practice. Focus Except. Child., 20, (1988).

31. Mercer, C. D. \& Miller, S. P. Teaching Students with Learning Problems in Math to Acquire, Understand, and Apply Basic Math Facts. Remedial Spec. Educ., 13, 19-35 (1992).

32. Westwood, P. The problem with problems: Potential difficulties in implementing problem-based learning as the core method in primary school mathematics. Aust. J. Learn. Difficulties, 16, 5-18 (2011).

33. Vanbinst, K., Ghesquière, P. \& De Smedt, B. Does numerical processing uniquely predict first graders' future development of single-digit arithmetic? Learn. Individ. Differ., 37, 153-160 (2015).

34. Maccini, P. \& Gagnon, J. C. Best practices for teaching mathematics to secondary students with special needs. Focus on Exceptional Children, 32(5) (2000).

35. Widaman, K. F., Little, T. D., Geary, D. C. \& Cormier, P. Individual differences in the development of skill in mental addition: Internal and external validation of chronometric models. Learn. Individ. Differ., 4, 167-213 (1992).

36. Campbell, J. L. D. Mechanisms of Simple Addition and Multiplication: A Modified Network-interference Theory and Simulation. Math. Cogn., 1, 121-164 (1995).

37. Bratina, T. \& Krudwig, K. Get It Right and Get It Fast! Building Automaticity to Strengthen Mathematical Proficiency. Focus Learn. Probl. Math., 25, 47 (2003).

38. Woodward, J. Developing automaticity in multiplication facts: Integrating strategy instruction with timed practice drills. Learning Disability Quarterly, 29 269-289 (2006). 
39. Baker, A. T. \& Cuevas, J. The Importance of Automaticity Development in Mathematics. Georg. Educ. Res., 14, 13 (2018).

40. Reigosa-Crespo, V., Valdés-Sosa, M., Butterworth, B., Estévez, N., Rodríguez, M., Santos, E., Torres, P., Suárez, R., \& Lage, A. Basic numerical capacities and prevalence of developmental dyscalculia: The Havana survey. Dev. Psychol., 48(1), 123-135 (2012).

41. Geary, D. C., Hoard, M. K. \& Bailey, D. H. Fact Retrieval Deficits in Low Achieving Children and Children With Mathematical Learning Disability. J. Learn. Disabil., 45, 291-307 (2012).

42. Jordan, N. C., Hanich, L. B. \& Kaplan, D. Arithmetic fact mastery in young children: A longitudinal investigation. J. Exp. Child Psychol., 85, 103-119 (2003).

43. Faul, F., Erdfelder, E., Lang, A. G. \& Buchner, A. G*Power 3: A flexible statistical power analysis program for the social, behavioral, and biomedical sciences. in Behavior Research Methods, 39 175-191 (2007).

44. Cohen, J. Statistical Power Analysis for the Behavioral Sciences (2013).

45. Tukey, J. Exploratory data analysis, 2, pp. 131-160 (1977).

46. Dotan, D. \& Friedmann, N. Reducing interference improves the memorization of multiplication facts in case of hypersensitivity to interference. J. Numer. Cogn., 5, 400-430 (2019).

47. Dotan, D. \& Zviran-Ginat, S. Elementary math in elementary school: To learn the multiplication table, avoid proactive interference. PsyArXiv (2021) doi:10.31234/OSF.IO/547A8.

48. Peirce, J., Gray, J.R., Simpson, S. et al. PsychoPy2: Experiments in behavior made easy. Behav. Res., 51, 195-203 (2019).

49. Katzoff, A., Zigdon, N. M. \& Ashkenazi, S. Difficulties in retrieval multiplication facts: The case of interference to reconsolidation. Trends Neurosci. Educ., 20, (2020).

50. Herszage, J. \& Censor, N. Modulation of Learning and Memory: A Shared Framework for Interference and Generalization. Neuroscience, 392, 270-280 (2018).

51. Gabitov, E., Boutin, A., Pinsard, B. et al. Re-stepping into the same river: Competition problem rather than a reconsolidation failure in an established motor 
skill. Sci. Rep., 7, 9406 (2017).

52. Shmuel, D., Frank, S. M., Sharon, H., Sasaki, Y., Watanabe, T., \& Censor, N. Early Visual Cortex Stimulation Modifies Well-Consolidated Perceptual Gains. Cereb. Cortex, 31, 138-146 (2021).

53. Herz, N., Bar-Haim, Y., Tavor, I., Tik, N., Sharon, H., Holmes, E. A., \& Censor, N. Neuromodulation of Visual Cortex Reduces the Intensity of Intrusive Memories. Cereb. Cortex, 32, 408-417 (2022).

54. Skagenholt, M., Träff, U., Västfjäll, D. \& Skagerlund, K. Examining the Triple Code Model in numerical cognition: An fMRI study. PLoS One, 13, e0199247 (2018).

55. Moeller, K., Willmes, K. \& Klein, E. A review on functional and structural brain connectivity in numerical cognition. Frontiers in Human Neuroscience, 9 (2015).

56. Nieder, A. The neuronal code for number. Nature Reviews Neuroscience, 17 366-382 (2016).

57. Dehaene, S. Varieties of numerical abilities. Cognition, 44, 1-42 (1992).

58. O’Neill, J., Pleydell-Bouverie, B., Dupret, D. \& Csicsvari, J. Play it again: reactivation of waking experience and memory. Trends Neurosci., 33, 220-229 (2010).

59. Karpicke, J. D. \& Roediger, H. L. The critical importance of retrieval for learning. Science, 319, 966-968 (2008).

60. Knowles, N. P. The relationship between timed drill practice and the increase of automaticity of basic multiplication facts for regular education sixth graders. Walden University (2010). 